\title{
Die Bestimmung des Chlorids in Plasma und Serum (Quecksilber [II]-Thiocyanat-Methode) mit dem Greiner Electronic Selective Analyzer GSA II
}

\author{
Von H. Küffer, R. Richterich $\left.{ }^{1}\right), R$. Kraft, E. Peheim und J. P. Colombo \\ Chemisches Zentrallaboratorium, Inselspital CH-3010 Bern, Schweiz und Greiner Electronic AG, \\ CH-4900 Langenthal, Schweiz
}

(Eingegangen am 15. März 1974/3. Februar 1975)

1. Eine Quecksilber [II]-Thiocyanat-Methode zur Bestimmung von Chlorid in Plasma und Serum wurde für den Greiner Electronic Selective Analyzer GSA II adaptiert. Durch Weglassen von Thiocyanat im Leerwert-Ansatz wird ein Proben- und partieller Reagenzien-Leerwert durchgeführt.

2. Der Reaktions-Ablauf wurde untersucht. Bei einer Reaktionszeit von 350-500 s liegt der lineare Meß-Bereich zwischen 30 und $130 \mathrm{mmol} / 1$. Zwischen 90 und $110 \mathrm{mmol} / \mathrm{l}$ beträgt die Abwcichung zwischen Ist-Wert und Soll-Wert weniger als $1 \%$.

3. Wahrscheinlich als Folge unterschiedlicher Verunreinigungen der Reagenzien mit Chlorid-Ionen muß der Eichfaktor kontrolliert und gegebenenfalls nachkalibriert werden.

4. Hämolyse, Lipämie und Bilirubin stören nicht. Das Vorliegen von Protein hat keinen Einfluß auf den Reaktionsablauf.

5. Bei Konzentrationen um $100 \mathrm{mmol} / 1$ beträgt die Präzision in der Serie, ausgedrückt als Variations-Koeffizient (\%), bei wäßrigen Lösungen $0,3-0,6 \%$, bei flüssigen Kontroll-Seren $0,4-0,8 \%$ und bei lyophilisierten Kontroll-Seren $0,8-1,5 \%$.

6. Eine Verschleppung von 150 auf $10 \mathrm{mmol} / \mathrm{l}$ ist nicht nachweisbar.

The determination of chloride in plasma and serum (mercury(II)-thiocyanate method) with the Greiner Electronic Selective Analyzer GSA II.

1. A mercury(II)-thiocyanate method for the determination of chloride in plasma and serum was adapted for the Greiner Electronic Selective Analyzer GSA II. A sample blank value and a partial reagent blank value were determined by omitting thiocyanate from the control system.

2. The course of the reaction was investigated. For a reaction time of $350-500 \mathrm{~s}$, the response was linear between 30 and $130 \mathrm{mmol} / \mathrm{l}$. Between 90 and $110 \mathrm{mmol} / 1$, the deviation between the actual and the theoretical value is less than $1 \%$.

3. The calibration must be checked and, if necessary, restandardized; this is probably due to variable contamination of the reagents with chloride ions.

4. Haemolysis, lipaemia and bilirubin do not interfere. Protein has no effect on the course of the reaction.

5. At concentrations around $100 \mathrm{mmol} / \mathrm{l}$, the in series precision, expressed as the variation coefficient (\%), is $0.3-0.6 \%$ for aqueous solutions, $0.4-0.8 \%$ for liquid control sera, and $0.8-1.5 \%$ for lyophilized control sera.

6. No carry over was detectable from samples containing 150 to those containing $10 \mathrm{mmol} / \mathrm{l}$.

Aus der Vielzahl der bekannten photometrischen Techniken zur Bestimmung des Chlorids hat sich bis heute in der Klinischen Chemie nur die Quecksilber [II]-Thiocyanat-Methode (Rhodanid-Methode) durchsetzen können. Iwasaki et al. (1) zeigten im Jahre 1952, daß in einem Reagenz das Quecksilber [II]-Thiocyanat, EisenIonen und Salpetersäure enthält, bei der Zugabe von Chlorid-Ionen Rhodanid-Ionen freigesetzt werden, die mit den Eisen-Ionen einen orangeroten Komplex bilden. Die Reaktion ist seither eingehend untersucht worden $(2,3)$ und gehört wegen ihrer hohen Empfindlichkeit zu den Standardmethoden der analytischen Chemie

1) Prof. Dr. R. Richterich starb am 25. Dezember 1973 während der Fertigstellung dieser Arbeit.
(4-7). Ihren Eingang in die Klinische Chemie fand die Methode im Jahre 1957 als sie von Skeggs (8) für den AutoAnalyzer (Technicon) adaptiert wurde $(9,10)$. Für die manuelle Analytik wurden ähnliche Methoden von Schoenfeld \& Lewellen (11) und von Hamilton (12) entwickelt. Clauss et al. (13) modifizierten die Technik für den Analysator C 4 (Perkin-Elmer).

Im Laufe unserer Methoden-Adaptationen für den Greiner Electronic Selective Analyzer GSA II (GSA II) gingen wir von den Arbeiten von Schoenfeld \& Lewellen (11) und Clauss et al. (13) aus. Dabei mußten eingehende Untersuchungen über die Optimierung der Reagenzien und der Prozeßß-Zeit, sowie über den Linearitäts-Bereich und mögliche Störfaktoren durchgeführt werden. Wir 
berichten in dieser Arbeit über die Resultate dieser Untersuchungen, sowie über die praktischen Erfahrungen mit dieser Methode während über eines Jahres.

\section{Material und Methoden}

Chemikalien

Eiscn [III]-Nitrat, Nonahydrat, p. a. (Merck Nr. 3883)

Kalium-Chlorid, puriss, p. a. (Fluka Nr. 60130)

Quecksilber [1I]-Chlorid, p. a. (Merck Nr. 4419)

Quecksilber [II]-Nitrat, p. a. (Merck Nr. 4434)

Quecksilber [II]-Thiocyanat, p. a. (Merck Nr. 4484)

Salpctersäure, 65 proz. $(14,50 \mathrm{~mol} / \mathrm{l})$ puriss., p. a. (Fluka

Nr. 84380)

\section{Quecksilbernitrat-Stamm-Lösung}

(18,5 mmol/l). $6 \mathrm{~g}$ Quecksilber [II]-Nitrat und $1 \mathrm{ml}$ Salpetersäure werden mit demin./dest. Wasser ${ }^{2}$ ) auf 1 Liter gelöst. Unbeschränkt haltbar.

\section{Gebrauchslösungen}

\section{$R-1$ : Thiocyanat-Reagenz}

(Konzentrationen, mmol/l: Eisen [III]-Nitrat 19,8, Quecksilber [II]-Chlorid 0,203, Quecksilber [II]-Nitrat 0,0740, Quecksilber [II]-Thiocyanat 2,05, Salpetersäure 29,0). $650 \mathrm{mg}$ Quecksilber [II]-Thiocyanat verden bci $50-70^{\circ} \mathrm{C}$ unter Rühren in etwa $900 \mathrm{ml}$ demin./dest. Wasser gelöst. Nach dem Abkühlen auf Raumtemperatur werden $2 \mathrm{ml}$ Salpetersäure, $55 \mathrm{mg}$ Quecksilber [II]-Chlorid und $8 \mathrm{~g}$ Eisen [ II]-Nitrat in dieser Reihenfolge zugegeben. Rühren, bis alles gelöst ist. $4 \mathrm{ml}$ Quecksilbernitrat-Lösung zugeben und mit demin./dest. Wasser auf 1 Liter verdünnen. Im Dunkeln und bei $4{ }^{\circ} \mathrm{C}$ mindestens $1 \mathrm{~J}$ ahr haltbar.

\section{R-2: Leerwert-Reagenz}

(Konzentrationen, mmol/1: Eisen [III]-Nitrat 19,8, Quecksilber [II]-Nitrat 0,0740, Salpetersäure 29,0). $2 \mathrm{ml}$ Salpetersäure und $8 \mathrm{~g}$ Eisen [III]-Nitrat werden in einigen $\mathrm{ml}$ demin./dest. Wasser gelöst. $4 \mathrm{ml}$ der Quecksilbernitrat-Lösung zugeben und mit demin./dest. Wasser auf 1 Liter auffüllen. Im Dunkeln und bei $4^{\circ} \mathrm{C}$ mindestens $1 \mathrm{Jahr}$ haltbar.

\section{Vorgehen}

Die Methode C CL 1 (Chemie, Chlorid, Methode 1) ist schematisch in Abbildung 1 dargestellt; es wird ein Proben- und partieller Reagenzien-Leerwert durchgeführt. Die Programmierung geht aus Abbildung 2 hervor.

2) Natürliches Wasser enthält je nach Art und geologischer Lage zwischen 50 und $1000 \mathrm{mg} / \mathrm{l}$ bzw. 1,5-30 mmol/1 Chlorid. Für Trinkwasser gilt in den USA eine maximal zulässige Konzentration von $250 \mathrm{mg} / \mathrm{l}$, bzw. $7 \mathrm{mmol} / \mathrm{l}$ (Wright C. V.: Public Health Repts (1962), 77, 628-632). Für „Reagent Grade Water" fordert die American Chemical Society (Reagent Chemicals, ec. (1961) Kenyon R. L. Amer. Chem. Soc., 3rd, ed., Washington D. C.) eine maximale ChloridKonzentration von $0,1 \mathrm{mg} / 1$, bzw. $2,8 \mu \mathrm{mol} / 1$. Eine solche Wasser-Qualität ist aber weder nach Ionen-Austausch noch nach Destillation mit Sicherzeit zu erreichen. Außer einem Carryover von Chlorid bei Destillation (Henry J. R., zit. and Winstead M: Reagent Grade Water (1967) Amer. Soc. Med. Technol., Austin Tex.: Steck), ist auch mit einer Kontamination aus der Luft zu rechnen (Bergmann J. G. \& Sanik J. (1957): Analyt. Chem. 29, 241-243). Wir empfehlen daher für die Herstellung der Reagenzien zur ChloridBestimmung sowie als Verdünnungs-Flüssigkeit (Dosierer Nr. 100, 99 und 97) Wasser zu verwenden, das zuerst entionisiert und anschließend quarz-destilliert wurde (demin:/ dest. Wasser). Die spezifische Leitfähigkeit sollte unter $0,2 \mu \mathrm{S}$ liegen.

\begin{tabular}{|c|c|c|c|c|c|c|}
\hline \multirow{2}{*}{\multicolumn{2}{|c|}{ REAGENT }} & \multicolumn{2}{|c|}{ DISPENSER } & \multicolumn{2}{|c|}{ VOLUME } & TUBE \\
\hline & & $s$ & nt & 1 & ॥I & \\
\hline & $S+D F(20+100)$ & $594 / 600$ & $99 / 100$ & 120 & 120 & \\
\hline & DF (120) & 582 & 97 & - & - & \\
\hline R.1 & Thiocyenate Reagent & 408 & 68 & - & 2500 & \\
\hline R.2 & Blank Roagent & 216 & 36 & 2500 & - & \\
\hline R.3 & & & & & & \\
\hline R.4 & . & & & ' & & \\
\hline
\end{tabular}

Fig. 1. Darstellung der Methode zur Bestimmung des Chlorids in Plasma und Serum (C CL 1) mit dem GSA II.

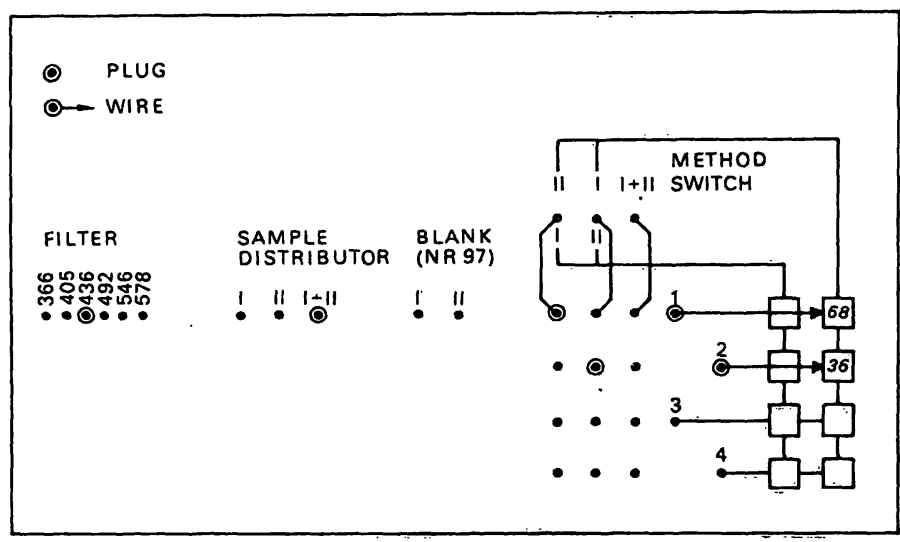

Fig. 2. Methoden-Programm C CL 1.

Konstanten

$\epsilon(436 \mathrm{~nm})=829,81 / \mathrm{cm} \cdot \mathrm{mol}$

Proben-Volumen (SV, sample volume) $=20 \mu \mathrm{l}$

End-Volumen (FV, final volume) $=2620 \mu$ !

Meß-Bedingungen

Im Leerwert-Ansatz I liegen die folgenden End-Konzentrationen vor (mmol/1): Eisen [III]-Nitrat 18,9, Quecksilber [II]-Nitrat 0,071, Salpetersäure 27,7, Chlorid 0-1,145. Der Proben-Ansatz II enthält zusätzlich: Quecksilber [II]-Chlorid 0,194, Quecksilber [II]-Thiocyanat 1,96. Die Reaktionszeit muß zwischen 350 und $500 \mathrm{~s}$ liegen.

Berechnung

$c=\mathrm{dA} \times \frac{1}{\epsilon} \times \frac{\mathrm{FV}}{\mathrm{SV}} \times 10^{3} \mathrm{mmol} / 1$

$\mathrm{c}=\mathrm{dA} \times 157,9 \mathrm{mmol} / \mathrm{l}$

$\mathrm{k}=1579$ (Methoden-Print)

$\mathrm{n}=1$ (Stellen nach dem Komma)

\section{Resultate und Diskussion}

Zur Optimierung der Reagenz-Zusammensetzung Stark vereinfacht spielen sich bei der Rhodanid-Methode die folgenden Reaktionen ab:

(1) $2 \mathrm{Cl}^{-}+\mathrm{Hg}(\mathrm{SCN})_{2}$

$$
\mathrm{HgCl}_{2}+2 S C N^{-}
$$

(2) $S C N^{-}+\mathrm{Fe}^{+++}$

$$
\mathrm{Fe}(\mathrm{SCN})^{++} \text {. }
$$

Zum Ablauf der Reaktion sind daher auf jeden Fall Quecksilber [II]-Thiocyanat-Ionen, Eisen-Ionen und Säure notwendig. 
Quecksilber [II]-Thiocyanat: Die meisten Autoren (1-12) verwendeten eine gesättigte wäßrige Lösung (Löslichkeit bei $25^{\circ} \mathrm{C}$ etwa $690 \mathrm{mg}$ im Liter, d. h. etwa $2,5 \mathrm{mmol} / \mathrm{l}$ ). Gesättigte Lösungen müssen in der absoluten Analytik vermieden werden, da sie schlecht reproduzierbar sind. Auch müßte beim GSA II, in dem die Reagenzien bei $4^{\circ} \mathrm{C}$ aufbewahrt werden, mit Ausfällungen gerechnet werden. Wir entschieden uns daher für die von Clauss et al. (13) gewählte Konzentration von $2 \mathrm{mmol} / \mathrm{l}$. Bei dieser Konzentration kommt es auf Grund unserer Erfahrung zu keinen Ausfällungen bei $4^{\circ} \mathrm{C}$. Da Plasma unter unseren Meßsbedingungen 131 fach verdünnt wird $(F V / S V=2620 / 20)$, liegt das Chlorid im Ansatz in Konzentrationen um 0,381 (entsprechend $50 \mathrm{mmol} / \mathrm{l}$ Probe), 0,763 $(100 \mathrm{mmol} / \mathrm{l})$ bis $1,15 \mathrm{mmol} / \mathrm{l}(150 \mathrm{mmol} / \mathrm{l})$ vor. Bei einer $1,96 \mathrm{mmol} / \mathrm{l}$ Quecksilber [II]-Thiocyanat-Lösung im Ansatz liegt somit ein genügender Überschuß für eine 1:1 Reaktion vor.

Eisen-Ionen: Die Eisen-Konzentration ist nicht kritisch, falls das Eisen im Überschuß vorliegt (12). Wird die Konzentration zu stark erhöht, so steigt auch der Leer-Wert entsprechend an. Übereinstimmend verwenden alle Autoren eine Konzentration zwischen 15 und $32 \mathrm{mmol} / \mathrm{l}$.

Säure: Da das Eisen-Salz ionisiert vorliegen muß, kann die Reaktion nur in saurem Milieu ablaufen. Die meisten Autoren $(1,2,8-11)$ verwenden Salpetersäure. Nur Zall (3) und Hamilton (12) bevorzugen Perchlorsäure, da diese eine höhere Absorbanz geben soll. Die Verwendung von Perchlorsäure bringt jedoch mehrere Nachteile mit sich. So werden die Proteine präzipitiert, was allerdings durch einen Zusatz von 3,3 mol/1 Harnstoff vermieden werden kann (12). Weiterhin wird der Reaktionsablauf stark von der Wasserstoffionen-Konzentration abhängig. Schließlich ist mit einer Reaktion des Choleste: rins mit dem in stark saurem Milieu vorliegende EisenSalz zu rechnen. Aus diesen Überlegungen entschieden wir uns zur Verwendung von Salpetersäure. Wie aus verschiedenen Untersuchungen hervorgeht, liegt die optimale Säure-Konzentration zwischen 20 und $30 \mathrm{mmol} / 1$.

Obwohl der Reaktionsablauf übersichtlich und einfach scheint, zeigten exakte Untersuchungen immer wieder, daß die Methode in dieser vereinfachten Form unbefriedigende Resultate gibt. So gilt bei allen bisher bekannten Modifikationen das Beer'sche Gesetz nur für einen sehr kleinen Meß-Bereich $(1,2,3,7,12)$; die meisten Autoren äußern sich zur Frage der Linearität überhaupt nicht $(8-11)$. Ebenfalls wurde immer wieder beobachtet, daß die Eichkurve nicht durch den Nullpunkt geht (1-3, 11, 12). Die Ursachen dieser Schwierigkeiten liegen sicher zum Teil in der stark variablen Chlorid-Kontamination der zur Reagenzien-Herstellung verwendeten Chemikalien und des Wassers. Es wurde sogar schon vermutet, daß selbst aus der Luft eine Kontamination mit Chlorid erfolgen kann (14). Eine gewisse Verbesserung läßt sich durch zwei Kunstgriffe erzielen: einem
Zusatz von Sublimat und von freien Quecksilber [II]Ionen.

Sublimat: Nach dem Massenwirkungs-Gesetz verschiebt zugesetztes Sublimat die Reaktion (1) nach links. Wie zuerst Hamilton (12) zeigte, läßt sich auf diese Weise die Empfindlichkeit der Mcthode herabsetzen, bzw. auf die gewünschte Stufe einstellen. Gleichzeitig verbessert ein Zusatz von Sublimat den Linearitätsbereich (12). Für unsere $Z$ wecke erwies sich eine Sublimat-Konzentration von $0,19 \mathrm{mmol} / \mathrm{l}$ als zweckmäßig.

Freie Quecksilber [II]-Ionen: Die Beobachtung, daß die Eich-Kurve meist nicht durch den Null-Punkt geht, dürfte in den meisten Fällen auf eine Verunreinigung durch Chlorid-Ionen zurückzuführen sein. Hamilton (12) zeigte, daßs es durch den Zusatz einer genau dosierten Menge Quecksilber [II]-Ionen, z. B. als Quecksilber [II]-Nitrat gelingt, die Eich-Kurve so zu verschieben, daß sie durch den Null-Punkt geht. Ein Teil der vorhandenen Chlorid-Ionen werden dabei als Quecksilber [II]Chlorid gebunden und reagieren nicht mehr mit dem Thiocyanat.

Das von uns gewählte Reagenz ist, wie aus Tabelle 1 hervorgeht, ähnlich demjenigen von Humilton (12), doch verwendeten wir Salpetersäure statt Perchlorsäure und konnten dadurch auf den Harnstoff-Zusatz verzichten.

Tab. 1. Zusammensetzung einiger gebräuchlicher Reagenzien für die Rhodanid-Methode.

\begin{tabular}{|c|c|c|c|c|}
\hline Komponenten & 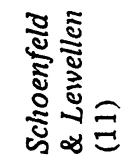 & 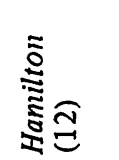 & 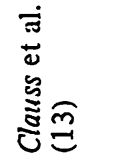 & $\stackrel{\underline{\underline{3}}}{\underline{\underline{O}}}$ \\
\hline $\mathrm{Hg}(\mathrm{SCN})_{2}[\mathrm{mmol} / \mathrm{l}]$ & 2,5 & 2,5 & 2,0 & 1,96 \\
\hline $\mathrm{Fe}\left(\mathrm{NO}_{3}\right)_{3}[\mathrm{mmol} / 1]$ & 32 & 15 & 20 & 18,9 \\
\hline Säure: Art & $\mathrm{HNO}_{3}$ & $\mathrm{HClO}_{4}$ & $\mathrm{HNO}_{3}$ & $\mathrm{HNO}_{3}$ \\
\hline $\begin{array}{l}\text { Konzentration } \\
{[\mathrm{mmol} / \mathrm{l}]}\end{array}$ & 25 & 4600 & 30 & 27,7 \\
\hline $\mathrm{HgCl}_{2}[\mathrm{mmol} / 1]$ & - & 0,6 & 0,75 & 0,19 \\
\hline $\begin{array}{l}\mathrm{Hg}^{++} \text {, als Nitrat } \\
{[\mathrm{mmol} / \mathrm{l}]}\end{array}$ & 0,9 & 0,3 & - & 0,071 \\
\hline Harnstoff [mmol/l] & - & 3300 & - & - \\
\hline$\epsilon[1 / \mathrm{cm} \cdot \mathrm{mol}]$ & - & 818 & 634 & 830 \\
\hline Proben-Verdünnung & 31 & 101 & 181 & 131 \\
\hline Proben-Volumen [ $\mu 1]$ & 500 & 100 & 15 & 20 \\
\hline
\end{tabular}

Optische Eigenschaften des Reaktions-Produktes

Beim vollständigen Ablauf unter geeigneten Bedingungen soll nur ein farbiges End-Produkt, $\mathrm{FeSCN}^{++}$, vorliegen (15). Für die Bildung eines 1:1 Komplexes sprechen Untersuchungen mit der Methode der „kontinuierlichen Variation" (16), sowie Beobachtungen über die Stabilität verschiedener Komplexe (15). Die Art und Zahl der Komplex-Species hängt jedoch sicher von den ReaktionsBedingungen (3) ab und dürfte auch während der einzelnen Phasen nicht konstant sein. 


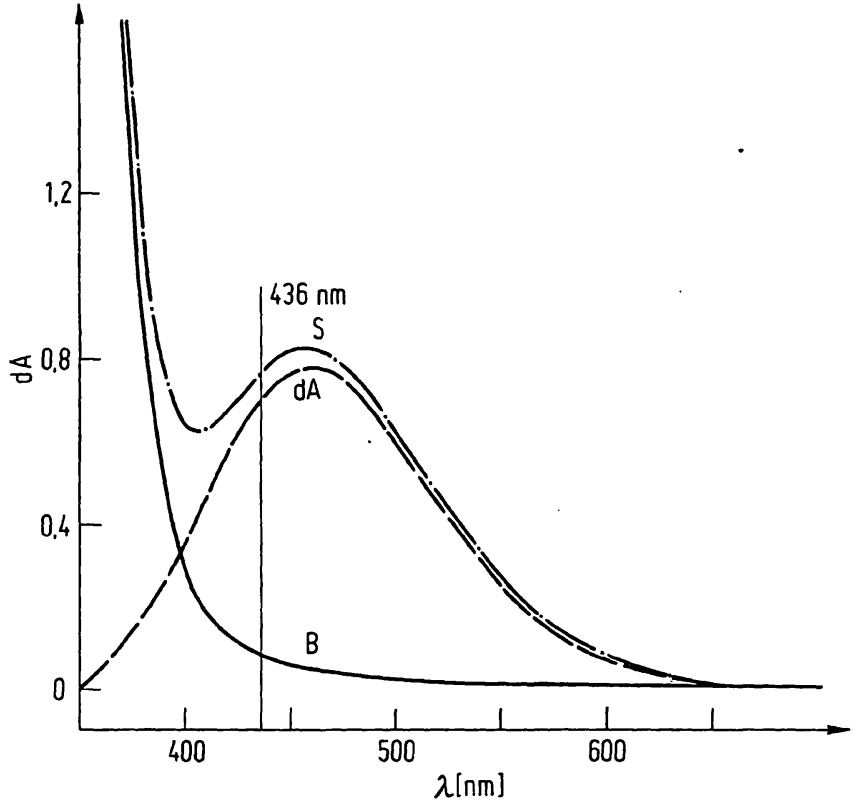

Fig. 3. Absorbanz des Proben-Ansatzes (S), des Leerwert-Ansatzes (B) und der Differenz (dA) als Funktion der Wellenlänge.

Die spektralen Eigènschaften des Reaktions-Produktes unter den vorliegenden Bedingungen sind in Abbildung 3 dargestellt. Die Absorbanz des Leerwertes ist im sichtbaren Bereich gering und fallt mit zunehmender Wellenlänge gegen Null. Das Maximum des Proben-Ansatzes liegt bei $450 \mathrm{~nm}$, dasjenige des Differenz-Spektrums bei $455 \mathrm{~nm}$. Clauss et al. (13) fanden das Maximum bei $460 \mathrm{~nm}$, Schoenfeld und Lewellen (11) in Perchlorsäure bei $480 \mathrm{~nm}$.

Bei der Adaption der Methode für den GSA II stellte sich die Frage, ob die Messung besser bei $436 \mathrm{~nm}$ oder bei $492 \mathrm{~nm}$ durchgeführt werden sollte. Theoretisch hätte die Messung bei $492 \mathrm{~nm}$ gewisse Vorteile, indem eine möglicherweise vorhandene Trübung sich weniger stark auswirken würde und zudem der Leerwert etwas kleiner sein dürfte (Abb. 3). Andererseits liegt die Absorbanz bei $492 \mathrm{~nm}$ etwa 20\% tiefer als bei $436 \mathrm{~nm}$. Experimentell konnten wir jedoch keine Unterschiede in Bezug auf die Abweichung vom Null-Punkt und den linearen Meßbereich feststellen. Wir geben daher der empfindlicheren Messung bei $436 \mathrm{~nm}$ den Vorzug.

Es sei hier noch erwähnt, daß die Absorbanz des Reaktions-Produktes eine Temperatur-Abhängigkeit aufweist: pro $10^{\circ} \mathrm{C}$ steigt die Absorbanz um 7-8\% (12). Es muß daher bei der Messung eine exakte Einhaltung der Temperatur gefordert werden.

\section{Zur Reaktions-Kinetik}

Nach der Festlegung der Reagenz-Zusammensetzung und der Meß-Wellenlänge wurde die Farbentwicklung als Funktion der Reaktionszeit untersucht. Durch Verschieben des Reagenz-Dosierers wurde die Reaktionszeit variiert. Ein typisches Experiment ist in Abbildung 4 dargestellt. Es zeigt sich, daß keine einfachen kinetischen

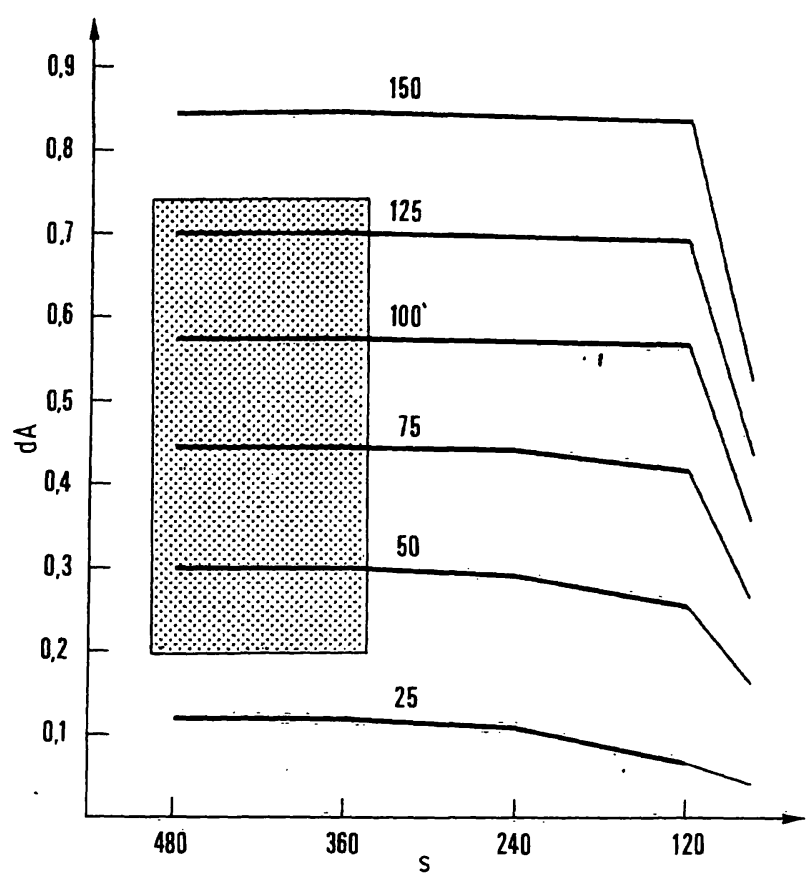

Fig. 4. Absorbanz als Furktion der Reaktionszeit (Zeit $0 \mathrm{~s}$ entspricht der Messung im Photometer) und der Chlorid-Konzentration (mmol/l). Der lineare MeßBereich ist hervorgehoben.

Verhältnisse vorliegen, sondern daß der Reaktionsablạuf offenbar u. a. durch die Chlorid-Konzentration beeinflußt wird. Bei relativ hohen Konzentrationen, etwa zwischen 100 und $150 \mathrm{mmol} / \mathrm{l}$ beträgt die Farb-Entwicklung bereits nach 120 s über $98 \%$. Mit abnehmender Chlorid-Konzentrationen verzögert sich die Reak= tion nicht nur absolut, sondern vor allem relativ.

Aus diesen Untersuchungen geht bereits hervor, daß

1. eine Linearität über den ganzen Meß-Bereich kaum erzielt werden kann und

2. Angaben über die Absorptivität immer nur für eine bestimmte Chlorid-Konzentration und ReaktionsDauer Gültigkeit haben.

\section{Die Gültigkeit deś Beer'schen Gesetzes}

Die folgenden Untersuchungen über den Meßbereich wurden mit Referenz-Material des National Bureau of Standards (Standard Reference Material 999, KaliumChlorid, Reinheit 99,9\%) durchgeführt. Das Material wurde bei $110^{\circ} \mathrm{C}$ bis zur Gewichts-Konstanz getrocknet und jede Verdünnung einzeln eingewogen. Die Reaktionszeit betrug bei allen Versuchen über $350 \mathrm{~s}$. Ein typisches Experiment ist in Tabelle 2 dargestellt. Offensichtlich geht die Kurve nicht durch den Null-Punkt und weicht auch sonst systematisch von einer Geraden ab. Hingegen zeigt sich, daß im Bereich zwischen 30 und $130 \mathrm{mmol} / 1$ die Abweichung weniger als $2 \%$ beträgt. Wir haben daher die Regression für diesen Bereich ermittelt und erhielten

$\mathrm{c}(\mathrm{mmol} / \mathrm{l})=-0,018+158,1 \mathrm{dA}$, 
Tab. 2. Gültigkeit des Beer'schen Gesetzes. $\mathrm{N}=10$.

\begin{tabular}{|c|c|c|c|c|}
\hline \multirow{2}{*}{$\begin{array}{l}\text { Chlorid, } \\
\text { eingewogen } \\
\text { [mmol/1] }\end{array}$} & \multicolumn{3}{|c|}{ Chlorid, gefunden } & \multirow{2}{*}{$\begin{array}{l}\begin{array}{l}\mathrm{Ab}- \\
\text { weichung }\end{array} \\
{[\mathrm{mmol} / \mathrm{l}]}\end{array}$} \\
\hline & $\begin{array}{l}\bar{x} \\
{[\mathrm{mmol} / \mathrm{l}]}\end{array}$ & $\begin{array}{l}\mathrm{s} \\
{[\mathrm{mmol} / \mathrm{l}]}\end{array}$ & $\begin{array}{l}\text { V. K. } \\
{[\%]}\end{array}$ & \\
\hline 0 & 1,68 & 0,2261 & 13,46 & $+1,68$ \\
\hline 10 & 4,23 & 0,3156 & 7,46 & $-5,77$ \\
\hline 20 & 15,07 & 0,2821 & 1,87 & $-4,93$ \\
\hline 30 & 28,00 & 0,5770 & 2,06 & $-2,00$ \\
\hline 40 & 40,09 & 0,4029 & 1,00 & $+0,09$ \\
\hline 50 & 51,81 & 0,4192 & 0,81 & $+1,81$ \\
\hline 60 & 62,48 & 0,2744 & 0,44 & $+2,48$ \\
\hline 70 & 72,34 & 0,5438 & 0,75 & $+2,34$ \\
\hline 80 & 82,17 & 0,6222 & 0,76 & $+2,17$ \\
\hline 90 & 91,45 & 0,4858 & 0,53 & $+1,45$ \\
\hline 100 & 100,02 & 0,4517 & 0,45 & $+0,02$ \\
\hline 110 & 109,00 & 0,5117 & 0,47 & $-1,00$ \\
\hline 120 & 118,03 & 0,3615 & 0,31 & $-1,97$ \\
\hline 130 & 127,39 & 0,7121 & 0,56 & $-2,61$ \\
\hline 140 & 136,52 & 0,6767 & 0,50 & $-3,48$ \\
\hline 150 & 145,59 & 0,5692 & 0,39 & $-4,41$ \\
\hline
\end{tabular}

mit einer Standard-Abweichung von $0,0525 \mathrm{mmol} / \mathrm{l}$ und einem Korrelations-Koeffizienten von 1,0 (5 Stellen). Eine graphische Darstellung der Abweichung von der Linearität gibt Abbildung 5. Zum Vergleich haben wir in dieser Abbildung auch noch die Abweichung eingezeichnet, wie sie mit dem Reagenz von Clauss et al. (13) erhalten wurde. Im klinisch wich tigsten Meßbereich, nämlich zwischen 90 und $110 \mathrm{mmol} / 1$ beträgt die $\mathrm{Ab}$ weichung weniger als $1 \%$.

Wie bereits oben erwähnt wurde, hängt die Absorbanz von mehreren Variablen ab, so von der Chlorid-Konzentration, der Prozeßzeit und noch anderen Faktoren wie vor allem Verunreinigungen durch Chlorid. Wir definieren daher in der vorliegenden Arbeit die molare Absorptivität willkürlich für eine Chlorid-Konzentration von $100 \mathrm{mmol} / \mathrm{l}$ und eine Prozeßzeit von 350 bis $500 \mathrm{~s}$

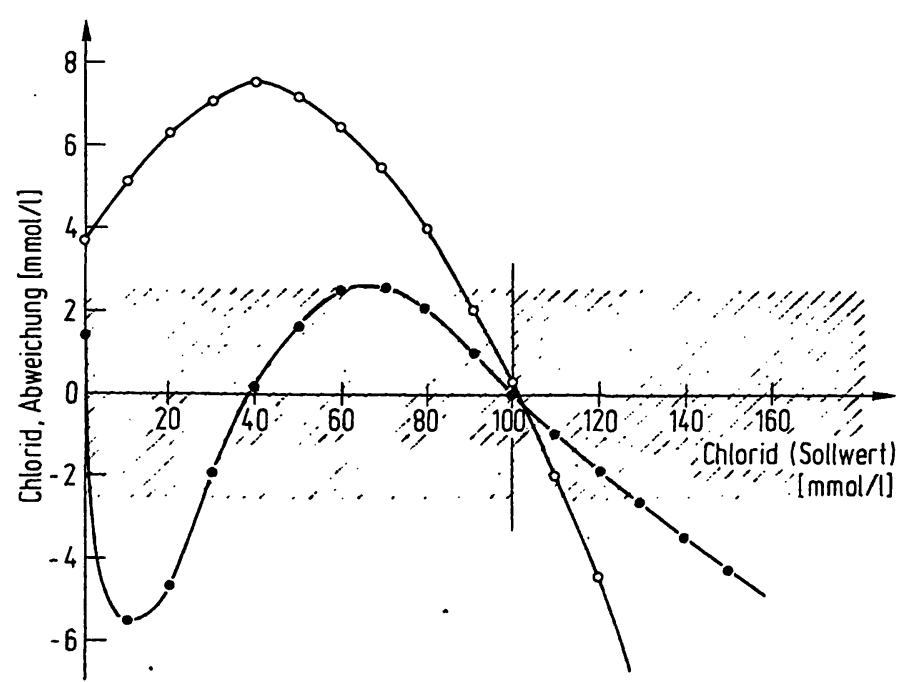

Fig. 5. Fchler-Darstellung der Methode. Auf der X-Achse ist die Soll-Konzentration in mmol/l dargestellt, auf der Y-Achse die Abweichung der.Meß-Werte von den Soll-Werten. Verwendet wurden die Daten aus Tab. 2. $\bigcirc-\circ$ Reagenz nach Clauss et al. (13), —— unter Methodik beschriebenes Reagenz. unter den Bedingungen des GSA II (Temperatur, Mischen, usw.). Unter diesen Einschränkungen fanden wir einen Wert von $829,8 \mathrm{l} / \mathrm{cm} \cdot \mathrm{mol}$. Im Vergleich dazu geben Clauss et al. (13) einen Wert von $634 \mathrm{l} / \mathrm{cm} \cdot \mathrm{mol}$ und Schoenfeld und Lewellen (11) von $.480 \mathrm{l} / \mathrm{cm} \cdot \mathrm{mol}$.

\section{Zur Wahl des Leerwertes}

Da bei der Chloridbestimmung mit einer Störung durch Lipämie, Hämolyse und Bilirubin zu rechnen ist $(12,13)$, muß auf jeden Fall ein Proben-Leerwert mitgeführt werden. Dabei drängt sich ein Reagenz auf, das kein Thiocyanat, jedoch Eisen-Ionen enthält. Es muß nämlich damit gerechnet werden, daß die große Zahl von Metaboliten und Medikamenten, die mit Eisen-Salzen reagieren $\left(\mathrm{FeCl}_{3}\right.$-Test) interferieren, falls keine Korrektur mit einem eisenhaltigen Leerwert vorgenommen wird. Aus ähnlichen Überlegungen wird auch beim AutoAnalyzer empfohlen, einen zweiten Kanal mit einem LeerwertReagenz einzusetzen (11).

\section{Spezifität}

Wie aus dem Prinzip der Methode hervorgeht, kann diese nicht spezifisch sein, sondern erfaßt neben Chlorid auch Bromid, Jodid, Fluorid und Cyanid $(1,11)$. Beim Cyanid, Fluorid und Jodid sind die Konzentrationen in Körperflüssigkeiten stets so gering, daß dies keine Rolle spielt. Hingegen kann es bei einer Überdosierung mit Bromiden zu falsch erhöhten Resultaten kommen; dies gilt allerdings auch für die meisten anderen im klinischen Laboratorium verwendeten Methoden. Wichtig ist vielleicht noch, darauf hinzuweisen, daß mit Fluoriden zur Glycolyse-Hemmung behandeltes Blut nicht für ChloridBestimmungen verwendet werden kann. Nur von theoretischem Interesse ist die Reaktion mit Thiosulfat, Ferrocyanid und Ferricyanid (2).

Eine positive Reaktion mit dem Rhodanid-Reagenz geben alle Metabolite und Medikamente, die eine positive Eisen-Chlorid-Reaktion geben, also u. a. Acetylsalicylat und $p$-Aminosalicylat (11). Das Mitführen eines Leerwertes eliminiert diese Störungen. Interferenzen sind ferner beim Vorliegen einer Hämolyse, Bilirubin und bei Trübungen beobachtet worden (11-13). Wie aus den Tabellen 3-5 hervorgeht, spielen diese Stör-Faktoren beim GSA II keine Rolle. Da bekannt ist, daß das Chlorid im Plasma zum Teil an Proteine gebunden vorliegt $(17,18)$, untersuchten wir auch den Effekt verschiedener Protein-Konzentrationen auf die Chlorid-Bestimmung. Wie Tabelle 6 zeigt, hat die Protein-Konzentration keinen Einfluß auf die Resultate der Chlorid-Bestimmung. Dies im Gegensatz zu Messungen mit ionenselektiven Elektroden.

\section{Beeinflussung}

Trotz mehrerer Versuche ist es uns mit den Methoden zur Bestimmung des Harnstoffs (19), der Glucose (20) und des Bilirubins (21) nie gelungen, beim GSA II eine 
Verschleppung nachzuweisen. Auch bei der Chlorid-Bestimmung verliefen Versuche mit einem KonzentrationsGefälle von 150 auf $10 \mathrm{mmol} / \mathrm{l}$ negativ.

Tab. 3. Störungen durch Hämolyse. Ein Erythrocyten-Konzentrat aus etwa $10 \mathrm{ml}$ Vollblut wurde mit demin. Wasser auf $5 \mathrm{ml}$ aufgefüllt. Nach Gefrieren und wieder Auftauen wurde das Präparat $10 \mathrm{~min}$ bei $15000 \mathrm{~g}$ zentrifugiert. Der Überstand wurde mit Seronorm (118) so verdünnt, daß Verdünnungen zwischen 21 und 1001 entstanden. $N=5$.

\begin{tabular}{|c|c|c|c|c|}
\hline \multicolumn{2}{|c|}{$\begin{array}{l}\text { Hämoglobin } \\
{[\mathrm{mmol} / \mathrm{l}][\mathrm{mg} / 100 \mathrm{ml}]}\end{array}$} & $\begin{array}{l}\text { Chlorid } \\
\frac{\mathrm{x}}{[\mathrm{mmol} / \mathrm{ll}}\end{array}$ & $\begin{array}{l}\mathrm{s} \\
{[\mathrm{mmol} / \mathrm{l}]}\end{array}$ & $\begin{array}{l}\text { V. K. } \\
{[\%]}\end{array}$ \\
\hline $\begin{array}{c}0 \\
12,4 \\
19,9 \\
24,8 \\
47,2 \\
70,1 \\
93,7 \\
117 \\
231 \\
287 \\
562\end{array}$ & $\begin{array}{r}0 \\
20 \\
32 \\
40 \\
76 \\
113 \\
151 \\
188 \\
373 \\
463 \\
905\end{array}$ & $\begin{array}{l}99,14 \\
98,64 \\
98,48 \\
99,00 \\
99,24 \\
98,58 \\
98,80 \\
98,42 \\
98,50 \\
98,24 \\
96,04\end{array}$ & $\begin{array}{l}0,833 \\
0,997 \\
1,543 \\
0,583 \\
0,522 \\
0,705 \\
0,529 \\
0,311 \\
0,561 \\
3,300 \\
1,071\end{array}$ & $\begin{array}{l}0,84 \\
1,01 \\
1,57 \\
0,59 \\
0,53 \\
0,72 \\
0,54 \\
0,32 \\
0,57 \\
3,36 \\
1,12\end{array}$ \\
\hline
\end{tabular}

Tab. 4. Beeinflussung der Chlorid-Bestimmung durch Bilirubin. $(N=4)$. Einc Verdünnungsreihe von Bilirubin (BDH) in $0,1 \mathrm{~mol} / 1 \mathrm{Na}_{2} \mathrm{CO}_{3}$ wurde als Lösungsmittel für Seronorm (Nr. 118) verwendet.

\begin{tabular}{llll}
\hline $\begin{array}{l}\text { Bilirubin } \\
{[\mu \mathrm{mol} / 1]}\end{array}$ & {$[\mathrm{mg} / 100 \mathrm{ml}]$} & $\begin{array}{l}\text { Chlorid } \\
{[\mathrm{mmol} / \mathrm{l}]}\end{array}$ & $\begin{array}{l}\mathrm{s} \\
{[\mathrm{mmol} / \mathrm{l}]}\end{array}$ \\
\hline 41,0 & 2,39 & 91,58 & 1,193 \\
54,9 & 3,21 & 91,88 & 0,613 \\
119,4 & 6,98 & 92,50 & 0,294 \\
199,2 & 11,65 & 92,13 & 0,880 \\
280,9 & 16,43 & 90,68 & 1,109 \\
355,6 & 20,82 & 91,63 & 0,607 \\
\hline
\end{tabular}

Tab. 5. Der Einflußs von Trübungen auf dic Chlorid-Bestimmungen. „Vor Klärung" heißt nach einer 5 min Zentrifugation bei $15000 \mathrm{~g}$, ,nach Klärung" nach einer 2,20 min Zentrifugation bei $15000 \mathrm{~g}$.

\begin{tabular}{|c|c|c|c|c|}
\hline Paticnt & $\begin{array}{l}\text { Triglyceride } \\
{[\mathrm{mg} / 100 \mathrm{ml}]}\end{array}$ & Cholesterin & $\begin{array}{l}\text { Chlorid } \\
\text { vor } \\
\text { Klärung } \\
\text { [mmol/1] }\end{array}$ & $\begin{array}{l}\text { nach } \\
\text { Klärung } \\
\text { [mmol/1] }\end{array}$ \\
\hline $\begin{array}{l}\text { S. V. } \\
\text { M. V. } \\
\text { S. O. } \\
\text { K. B. } \\
\text { L. W. } \\
\text { S. A. } \\
\text { P. E. } \\
\text { T. D. } \\
\text { B. E. } \\
\text { S. E. }\end{array}$ & $\begin{array}{r}420 \\
307 \\
458 \\
990 \\
1812 \\
585 \\
3510 \\
981 \\
390 \\
303\end{array}$ & $\begin{array}{r}268 \\
179 \\
298 \\
386 \\
555 \\
256 \\
323 \\
248 \\
1028 \\
324\end{array}$ & $\begin{array}{r}85,1 \\
91,1 \\
94,4 \\
104,2 \\
94,5 \\
86,4 \\
96,3 \\
89,2 \\
98,0 \\
101,2\end{array}$ & $\begin{array}{l}86,6 \\
88,5 \\
98,8 \\
98,8 \\
96,8 \\
87,4 \\
99,9 \\
89,7 \\
97,9 . \\
98,8\end{array}$ \\
\hline
\end{tabular}

Tab. 6. Einfluß der Protein-Konzentration auf die Chlorid-Bcstimmung. Es wurde zunächst eine Lösung mit einer Albumin-Konzentration von $100 \mathrm{~g} / \mathrm{l}$ hergestellt. Anschließend wurde Kalium-Chlorid eingewogen und in dicser Lösung gelöst. $N=10$. Die angeführten Sollwerte wurden auf dic Nicht-Lincarität korrigiert. $\mathrm{N}=10$.

\begin{tabular}{|c|c|c|c|c|}
\hline \multirow{2}{*}{$\begin{array}{l}\text { Chlorid, } \\
\text { eingewogen } \\
{[\mathrm{mmol} / \mathrm{l}]}\end{array}$} & \multirow{2}{*}{$\begin{array}{l}\text { Chlorid, } \\
\text { Soll-Wert } \\
\text { [mmol/1] }\end{array}$} & \multicolumn{3}{|c|}{$\begin{array}{l}\text { Chlorid, } \\
\text { mit Protein-Zusatz }\end{array}$} \\
\hline & & $\begin{array}{l}\bar{x} \\
{[\mathrm{mmol} / 1]}\end{array}$ & s & $\begin{array}{l}\text { V. K. } \\
{[\%]}\end{array}$ \\
\hline 0,0 & 0,17 & 0,18 & 0,0917 & 50,9 \\
\hline 20,0 & 15,07 & 14,44 & 0,6044 & 4,19 \\
\hline 45,0 & 45,95 & 44,48 & 0,6797 & 1,53 \\
\hline 67,5 & 69,43 & 69,88 & 0,7326 & 1,04 \\
\hline 90,0 & 91,45 & 91,86 & 0,6340 & 0,69 \\
\hline 112,5 & 111,72 & 111,76 & 0,7032 & 0,63 \\
\hline 135,0 & 131,95 & 131,93 & 0,5726 & 0,39 \\
\hline 150,0 & 145,59 & 144,98 & 0,3844 & 0,29 \\
\hline
\end{tabular}

\section{Rich tigkeit}

Die Richtigkeit der Methode wurde durch eine Analyse einer Reihe kommerzieller Kontroll-Seren überprüft. Einige Resultate sind in Tabelle 7 dargestellt. Die Resultate lagen zum größten Teil zwischen 98 und $102 \%$ der deklarierten Werte, die Variations-Koeffizienten schwanken zwischen 0,44 und $1,16 \%$.

\section{Präzision}

Wie schon bei früheren Untersuchungen (21), läßt sich auch hier deutlich ein Specimen-Fehler nachweisen. Die kleinsten Streuungen werden auch bei der Chlorid-Bestimmung mit wäßrigen Lösungen beobachtet (vgl. Tab. 2 u. a.); der Variations-Koeffizient lag zwischen 0,4 und $0,8 \%$. Bei gelösten Kontroll-Seren liegen die entsprechenden Werte zwischen 0,6 und $1,2 \%$, während sie bei lyophilisierten Specimen zwischen 0,8 und 1,5\% liegen. Auf die grundsätzliche Bedeutung dieser Beobachtung haben wir schon früher hingewiesen.

Die Präzision aller bisher mit dem GSA II untersuchten Methoden folgt, wie auch aus Abbildung 6 hervorgeht, bestimmten Gesetzmäßigkeiten:

1. Die Standard-Abweichung $s$ ist eine Funktion der Absorbanz. Sie ist am kleinsten bei Absorbanz Null und steigt mit zunehmender Absorbanz, bzw. Konzentration.

2. Der Variations-Koeffizient ist umgekehrt bei einer Absorbanz von Null am grölsten und fällt mit steigender $\mathbf{A b}$ sorbanz gegen einen Grenzwert. Theoretisch sollte der Variations-Koeffizient bei Null unendlich groß sein. Die relativ kleinen Variations-Koeffizienten beim Nullwert bei der Bestimmung des Chlorids sind darauf zurïckzuführen, daß die Eich-Kurve nicht exakt durch Null geht.

\section{Praktische Erfahrungen}

Die vorliegende Methode wird seit dem Februar 1973 routinemäßig in unserem Laboratorium durchgeführt, wobei wir täglich etwa 100 Chlorid-Bestimmungen 
Tab. 7. Bestimmung der Chlorid-Konzentrationen in einigen kommerziellen Kontroll-Specimen $(N=10)$. L lyophilisiert, $F$ flüssig.

\begin{tabular}{|c|c|c|c|c|c|c|c|c|}
\hline \multirow[t]{2}{*}{ Specimen } & \multirow[t]{2}{*}{ Art } & \multicolumn{3}{|l|}{ Deklarierte Werte } & \multicolumn{3}{|c|}{ Gefundene Werte } & \multirow{2}{*}{$\frac{\text { Gefunden }}{\text { Deklariert }} \cdot 100$} \\
\hline & & Methode & $\begin{array}{l}\bar{x} \\
{[\mathrm{mmol} / \mathrm{ll}]}\end{array}$ & $\begin{array}{l} \pm 2 \mathrm{~s} \\
{[\mathrm{mmol} / 1]}\end{array}$ & $\begin{array}{l}\bar{x} \\
{[\mathrm{mmol} / \mathrm{l}]}\end{array}$ & $\stackrel{\mathrm{s}}{[\mathrm{mmol} / \mathrm{l}]}$ & $\begin{array}{l}\text { V. K. } \\
{[\%]}\end{array}$ & \\
\hline $\begin{array}{l}\text { Kontroll-Scrum } \\
\text { (C 0431) }\end{array}$ & $\mathrm{F}$ & Titrimetrie (1) & 98,0 & & 93,61 & 0,519 & 0,554 & 95,52 \\
\hline $\begin{array}{l}\text { Lab-Trol } \\
\text { (LT-40 A-Z) }\end{array}$ & $\mathrm{F}$ & $\begin{array}{l}\text { Titrimetrie (1) } \\
\text { Potentiometrie (2) } \\
\text { Auto-Analyzer (4) }\end{array}$ & $\begin{array}{r}100,7 \\
99,9 \\
99,4\end{array}$ & $\begin{array}{c}100,01-101,39 \\
98,8-101,0 \\
97,3-101,5\end{array}$ & 99,14 & 0,698 & 0,704 & $\begin{array}{l}98,45 \\
99,24 \\
99,74\end{array}$ \\
\hline $\begin{array}{l}\text { Moni-Trol II } \\
\text { (PTD-32, A, B) }\end{array}$ & $\mathbf{L}$ & $\begin{array}{l}\text { Titrimetrie (1) } \\
\text { Potentiometrie (2) } \\
\text { Auto-Analyzer (4) } \\
\text { SMA } 12 / 60\end{array}$ & $\begin{array}{l}114,6 \\
113,8 \\
114,6 \\
113,0\end{array}$ & $\begin{array}{l}113,0-116,2 \\
112,9-114,7 \\
112,5-116,5 \\
108,4-117,6\end{array}$ & 113,55 & 1,312 & 1,155 & $\begin{array}{r}99,08 \\
99,78 \\
99,17 \\
100,49\end{array}$ \\
\hline $\begin{array}{l}\text { Precinorm S } \\
(102)\end{array}$ & $\mathbf{F}$ & $\begin{array}{l}\text { Titrimetrie (1) } \\
\text { Potentiometrie (2) } \\
\text { Coulometrie (3) }\end{array}$ & $\begin{array}{r}103,0 \\
99,0 \\
99,3\end{array}$ & $\begin{array}{l}97,9-108 \\
94,1-104 \\
95,3-103\end{array}$ & 99,83 & 0,482 & 0,483 & $\begin{array}{r}96,92 \\
100,84 \\
100,53\end{array}$ \\
\hline $\begin{array}{l}\text { Q-Pack } \\
(0369 \mathrm{R} 002 \mathrm{Al})\end{array}$ & $\mathbf{F}$ & $\begin{array}{l}\text { Titrimetrie (1) } \\
\text { Potentiometrie (2) } \\
\text { SMA } 12 / 60\end{array}$ & $\begin{array}{l}104 \\
104 \\
104\end{array}$ & $101-107$ & 104,01 & 0,544 & 0,523 & $\begin{array}{l}100 \\
100 \\
100\end{array}$ \\
\hline Seronorm (120) & $\mathrm{L}$ & $\begin{array}{l}\text { Neutronen-Aktivierung } \\
\text { Potentiometrie (2) } \\
\text { Auto-Analyzer (4) }\end{array}$ & $\begin{array}{l}102,8 \\
100,9 \\
101,0\end{array}$ & & 102,57 & 0,473 & 0,461 & $\begin{array}{r}99,78 \\
101,66 \\
101,55\end{array}$ \\
\hline $\begin{array}{l}\text { Versatol-Pediatric } \\
(0586060)\end{array}$ & $\mathbf{L}$ & $?$ & 101,0 & -104 & 98,07 & 0,432 & 0,440 & 97,10 \\
\hline
\end{tabular}

(1) Titrimetrie: Schales, O. \& Schales, S. S. (1941), J. Biol. Chem. 140, 879.

(2) Potentiometrie: Seligson, D., McCormick, G. J. \& Sleeman, K. (1958), Clin. Chem. 4, 159.

(3) Coulometrie: Cotlove, E., Trantham, H. V. \& Bowman, R. L. (1958), J. Lab. Clin. Med. 51, 461.

(4) Auto-Analyzer (N-5b I/II): Zall, D. M., Fischer, D. \& Garner, M. O. (1956), Analyt. Chem. 28, 1665.

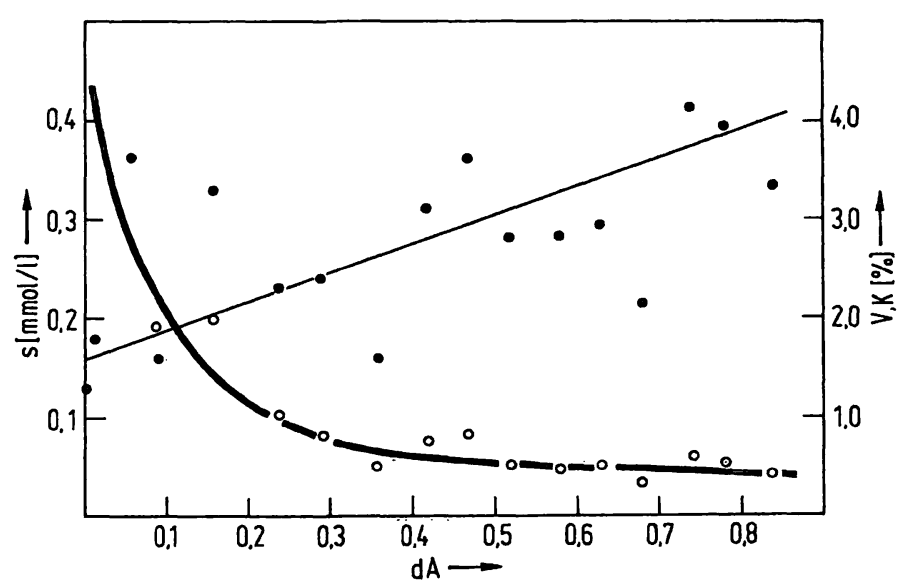

Fig. 6. Präzision der Chlorid-Bestimmung. Graphische Darstellung der Daten aus Tab. 2. Ordinate links: Standard-

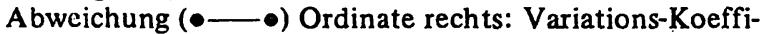
zient $(0-0)$ in $\%(n=10)$. Abszisse: Differenz der Absorbanz zwischen Proben- und Leerwert-Ansatz (dA).

durchführen. Einige Daten über die Repetierbarkeit und Reproduzierbarkeit der Methode sind in Tabelle 8 zusammengefaßt. Wie aus diesen Daten hervorgeht, lagen die Variations-Koeffizienten bei diesem lyophilisierten Kontroll-Serum zwischen 0,4 und 1,4\%, wobei kein Unterschied zwischen der Streuung in Serie (Repetierbarkeit) und der Streuung von Tag zu Tag (Reproduzierbarkeit) nachzuweisen ist.

Ein praktisch wichtiges Problem ist die Gefahr einer Kontamination der Reagenzien mit Chlorid. Dies tritt
Tab. 8. Resultate der Qualitäts-Kontrolle während einer Woche (Reproduzierbarkeit). Täglich wurde ein neues Fläschchen Monitrol II (Dade) gelöst und 10 Bestimmungen durchgeführt (Repetierbarkeit).

\begin{tabular}{|c|c|c|c|c|}
\hline & & $\begin{array}{l}\overline{\mathrm{X}} \\
{[\mathrm{mmol} / \mathrm{l}]}\end{array}$ & $\begin{array}{l}\mathrm{s} \\
{[\mathrm{mmol} / \mathrm{l}]}\end{array}$ & $\begin{array}{l}\text { V. K. } \\
{[\%]}\end{array}$ \\
\hline \multirow[t]{2}{*}{$\begin{array}{l}13.10 \\
15.10 \\
16.10 \\
17.10 \\
18.10 \\
19.10\end{array}$} & & $\begin{array}{l}110,0 \\
111,8 \\
109,8 \\
108,5 \\
110,0 \\
111,5\end{array}$ & $\begin{array}{l}1,50 \\
0,95 \\
1,04 \\
0,42 \\
1,29 \\
0,43\end{array}$ & $\begin{array}{l}1,37 \\
0,85 \\
0,95 \\
0,40 \\
1,18 \\
0,39\end{array}$ \\
\hline & Total & 110,26 & 1,210 & 1,09 \\
\hline
\end{tabular}

vor allem dann auf, wenn beim Einfüllen neuer Reagenzien-Flaschen der Stutzen mit bloßen Händen verschmutzt wird. Es müssen daher beim Reagenz-Wechsel stets Kunststoff-Handschuhe getragen werden. Grobe Verunreinigungen dieser Art lassen sich meist an der Verfärbung des Reagenz erkennen. Sie manifestieren sich auch sofort in einer starken Erhöhung der Werte bei der Null-Kontrolle.

\section{Rekalibrierung beim GSA II}

Bei den bisher für den GSA II publizierten Methoden zur Bestimmung des Harnstoffs (19), der Glucose (20) und des Bilirubins (21) war es nie notwendig, nach der InitialEichung (Methoden-Print) eine Rekalibrierung vorzuneh- 
men. Wir berichteten im März 1973, daß dies auch beim Chlorid nicht notwendig sei (22). Nach einigen Monaten zeigte sich jedoch, daß sehr langsam ein Drift auftreten kann, der eine Rekalibrierung notwendig macht. Zur Drift-Kontrolle muß allerdings eine wäßrige Chlorid-Lösung mitgeführt werden, da die Streuung der kommerziellen Kontroll-Seren zur Überwachung dieser Funktion zu groß ist. Wir betrachteten eine, Abweichung von $\pm 1 \%$ vom Soll-Wert ( $100 \mathrm{mmol} / \mathrm{l})$ als Alarm-Grenze, eine Abweichung von 1,5\% als Aktions-Grenze. Bei kommerziellen Kontroll-Seren, vor allem bei lyophilisierten Specimen, liegen solche Schwankungen in Form des Specimen-Fehlers vor, so daß diese nicht zu Kontroll-Zwekken verwendet werden dürfen. Es empfiehlt sich daher, täglich bei der Qualitäts-Kon trolle neben der Null-Kontrolle noch eine wäßrige Lösung bekannter Konzentration mitzu führen. Eine weitergehende Qualitäts-Kontrolle erübrigt sich. Die Drift-Abweichungen lassen sich schon früh erkennen. Wir mußten bisher etwa einmal pro Monat eine Rekalibrierung vornehmen. Das Prinzip der Rekalibrierung sei kurz erläutert.

\section{Es seien}

f der Faktor, mit dem die Absorbanz multipliziert wird, um eine Konzentration zu erhalten,

k die Methoden-Konstante, die als Hardware im MethodenPrint (gedruckte Schaltung) vorliegt,

n die Zahl der Stellen nach dem Komma, die durch den Vordruck auf der Bestell-Karte gegeben sind und

$v$ eine Variable, die dem Quotienten aus Erwartungs-Wert $\left(C_{\text {exp }}\right)$ und Beobachtungs-Wert $\left(C_{\text {obs }}\right)$ entspricht und am. Potentiometer des Methoden-Prints eingestellt werden kann.

Die Berechnung des Resultates erfolgt beim GSA II nach folgender Formel:

$c=d A \times f$, wobei

$\mathrm{f}=\mathrm{k} \times \mathrm{v} \times 10^{-\mathrm{n}}$.

Ist $v$ gleich $1, d$. h. Erwartungs-Wert/Beobachtungs-Wert $=1$, so beträgt die Potentiometer-Einstellung 400 Skalenteile. Weicht der Beobachtungs-Wert signifikant vom Erwartungs-Wert ab, so berechnet man, wie dies Tabelle 9 zeigt, v. Mit Hilfe einer Formel oder von Tabellen kann auf Grund der bekannten alten Skalen-Einstellung und des v die neue Skalen-Einstellung ermittelt werden.
Tab. 9. Auszug aus der Qualitäts-Kontrolle. Es sind nur diejenigen Daten angefüht, an denen eine Re-Kalibrierung notwendig war.

\begin{tabular}{|c|c|c|c|c|c|}
\hline Datum & $\begin{array}{l}\text { Erwartungs- } \\
\text { Wert } \\
C_{\text {exp }} \\
{[\mathrm{mmol} / \mathrm{l}]}\end{array}$ & $\begin{array}{l}\text { Beobach- } \\
\text { tungs-Wert } \\
\text { Cobs } \\
{[\mathrm{mmol} / 1]}\end{array}$ & $\begin{array}{l}v \\
\frac{C_{\text {exp }}}{C_{\text {obs }}}\end{array}$ & $\begin{array}{l}\text { Potentio- } \\
\text { meter } \\
\text { Skalen- } \\
\text { Teile }\end{array}$ & $\begin{array}{l}\text { Faktor } \\
\text { f }\end{array}$ \\
\hline $\begin{array}{l}16.4 . \\
17.5 . \\
24.8 . \\
21.9 . \\
2.10 .\end{array}$ & $\begin{array}{l}100,0 \\
100,0 \\
100,0 \\
100,0 \\
100,0\end{array}$ & $\begin{array}{r}100,0 \\
98,6 \\
101,5 \\
102,5 \\
98,4\end{array}$ & $\begin{array}{l}1,000 \\
1,024 \\
0,986 \\
0,975 \\
1,028\end{array}$ & $\begin{array}{l}400 \\
491 \\
434 \\
343 \\
452\end{array}$ & $\begin{array}{l}157,9 \\
161,7 \\
159,4 \\
155,4 \\
159,8\end{array}$ \\
\hline
\end{tabular}

Durch die neue Skalen-Einstellung am Potentiometer wird v wieder gleich 1 . Diese Korrektur kann in 1-2 min vorgenommen werden.

Die Ursachen für diese unsystematischen Schwankungen konnten wir bisher noch nicht ermitteln. Sie liegen aber vermutlich bei Chlorid-Verunreinigungen der Chemikalien, des Wassers und möglicherweise sogar Luft (14). Der Drift tritt sehr langsam ein und es war uns nur selten möglich, einen direkten Zusammenhang zwischen der Reagenzien-Charge und den Abweichungen festzustellen. Es scheint nicht ausgeschlossen, daß es durch eine Verbesserung der Reagenzien-Herstellung und Aufbewahrung gelingt, diese Fehlerquelle zu eliminieren.

Immerhin muß darauf hingewiesen werden, daß diese Abweichungen nur erkannt werden können, weil der GSA II eine außerordentlich hohe apparative Präzision aufweist. Bei Instrumenten, bei denen die apparative Präzision oberhalb derjenigen der Kontroll-Specimen liegt, d. h. über $1,5 \%$ beträgt, sind solche Veränderungen gar nicht feststellbar.

\section{Danksagung}

Wir danken Frl. H. Dauwalder, Frl. M. Rakebrandt, Frl. M. Lehmann und Herrn Dr. C. Tschanz für ihre wertvolle Mitarbeit bei der Ausarbeitung dieser Methode.

\section{Literatur}

1. Iwasaki, I., Utsumi, S. \& Ozana, T. (1952), Bull. Chem. Soc. Japan 25, 226.

2. Iwasaki, I., Utsumi, S., Hagino, K. \& Ozawa, T. (1956), Bull. Chem. Soc. Japan 29, 860-864

3. Zall, D. M., Fischer, D. \& Garner, M. Q. (1956), Anal. Chem. 28, 1665-1668.

4. Astm Standards, Part we, (Nov. 1969), Water, atmospheric analy sis, p. 29-31.

5. Demitt, T. F. (1962), U. S. A. E. C. rept. HW-74333.
6. Florence, T. M. \& Farrar, Y. J. (19711), Anal. Chim. Acta 54, 373-377.

7. Rodabaugh, R. D. \& Upperman, G. T. (1971), Anal. Chim. Acta 54, 373-377.

8. Skeggs, L. T. (1957), Amer. J. Clin. Pathol. 28, 311.

9. Skeggs, L. T. \& Hochstrasser, H. (1964), Clin: Chem. 10, 918.

10. Kenny, M. A. \& Cheng, M. H. (1964), Clin. Chem. 18, 352-354. 
11. Schoenfeld, R. G. \& Lewellen, C. J. (1964), Clin. Chem. 10, 533-539.

12. Hamilton, R. H. (1966), Clin. Chem. 12, 1-17.

13. Clauss, I., Graf, E., Ringhardtz, I. \& Schmitt, A. (1969), Beitr. z. klin. Chemie, Heft 5, (Bodenseewerk Perkin-Elmer).

14. Bergmann, J. G. \& Sanik, J. (1957), Anal. Chem. 29, 241243.

15. Elsheimer, H. N. \& Kochen, R. L. (1966), Anal. Chem. 38, 145-147.

16. Gould, R. K. \& Vosburgh, W. C. (1942), J. Amer. Chem. Soc. $69,1630$.
17. Scatchard, G., Schcinberg, H. I. \& Armstrong, S. H. (1950), J. Amer. Chem. Soc. 72, 535-542.

18. Dahms, H., Rock, R. \& Seligson, D. (1968), Clin. Chem. 14, 859-970.

19. Richterich, R. \& Küffer, H. (1973), diese Z. 11, 553-564.

20. Richterich, R., Küffer, H. \& Lorenz Erica, (1974), diese $Z$. $12,5-13$

21. Küffer, H., Richterich, R., Peheim, E. \& Colombo, J. P. (1974), diese Z. 12, 294-302.

22. Richterich, R. (1973), Aerztl. Lab. 19, 300-305.

Dipl.-Biochem. H. Küffer Biochemistry

Research \& Development

Greiner Electronic Ltd.

CH-4900 Langenthal

Switzerland 
